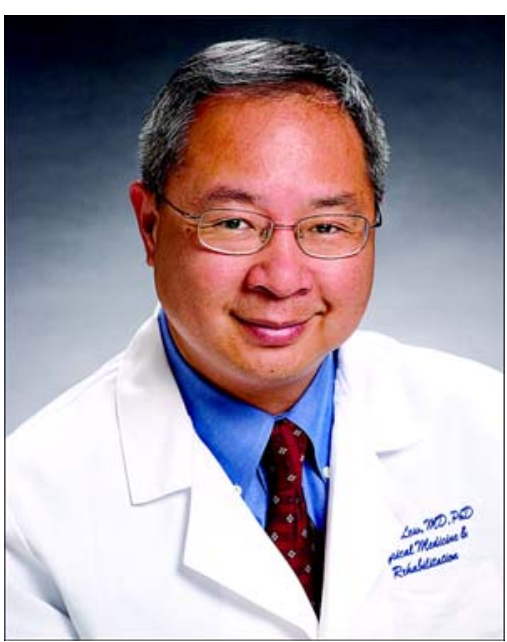

Henry L. Lew, MD, PhD; Coauthors include: John H. Poole, PhD; Sylvia B. Guillory, BS; Rose Marie Salerno, RN; Gregory Leskin, PhD; Barbara Sigford, MD, PhD

\section{Persistent problems after traumatic brain injury: The need for long-term follow-up and coordinated care}

Traumatic brain injury (TBI) is one of the leading causes of death and long-term disability in the United States [1]. Survivors of TBI experience various problems, including physical, cognitive, emotional, and community integration issues. Established in 1992, the Defense and Veterans Brain Injury Center (DVBIC) coordinates nine healthcare centers - two civilian, three military, and four Department of Veterans Affairs (VA) sites - that provide evidence-based treatment, education, and research on TBI (www.dvbic.org). Patients with TBI who are admitted to regional medical centers within the DVBIC network receive multidisciplinary assessment and rehabilitation by experts in physiatry (physical medicine and rehabilitation [PM\&R]), neurology, neuropsychology, psychiatry, and other allied health professions. After discharge, DVBIC patients are also advised to return for onsite, 3-day comprehensive follow-up evaluations at 1 and 2 years postinjury.

To determine the prevalence of a constellation of problems faced by the TBI patients admitted to our Palo Alto VA facility, we performed an extensive chart review on 138 patients who had sustained closed head injuries. These patients were enrolled in the DVBIC program at the Palo Alto VA from 1993 to 2003 and ranged in age from 18 to 76 (median $=27$ ). Of these patients, 71 percent returned for either the 1 or 2 year follow-up at the Palo Alto site and 49 percent returned for both follow-ups. Compared with the patients who returned for both follow-ups, those who missed one or both follow-ups had more emotional symptoms at baseline (mean $=3.2$ vs 2.4 symptoms/patient, $p<0.005$ ) but fewer cognitive impairments (4.2 vs 5.9 impairments/patient, $p<0.001$ ). The two groups did not differ significantly in age or the frequency of physical symptoms at baseline (4.4 vs 4.6, $p=0.5$ ).

The present analysis focused on those who returned for both follow-ups. We evaluated the patients' problems in four areas: physical, cognitive, emotional, and community integration, using standardized neurocognitive tests and structured clinical interviews. Physical problems were mainly documented by the physiatrist and included pain, motor weakness, gait abnormality, seizure, dizziness, and fatigue. Cognitive deficits were primarily measured by the neuropsychologist and included deficits in attention/concentration, processing speed, memory, problem-solving, executive organization, and safety judgment. Emotional issues, mainly identified by the psychiatrist, included depressed mood, anxiety, posttraumatic distress, suicidal ideation, irritability, and disinhibition. Community integration issues, primarily evaluated by the occupational therapist, included problems with self-care, money management, employment, community accessibility, recreational activities, and adjustment to limitations.

As Figure 1 demonstrates, 90 percent or more of TBI patients had at least one problem in each category at baseline, i.e., during the first week of their inpatient admission for acute rehabilitation. During the following 2 years, the frequency of physical problems decreased from 100 to 84 percent, 


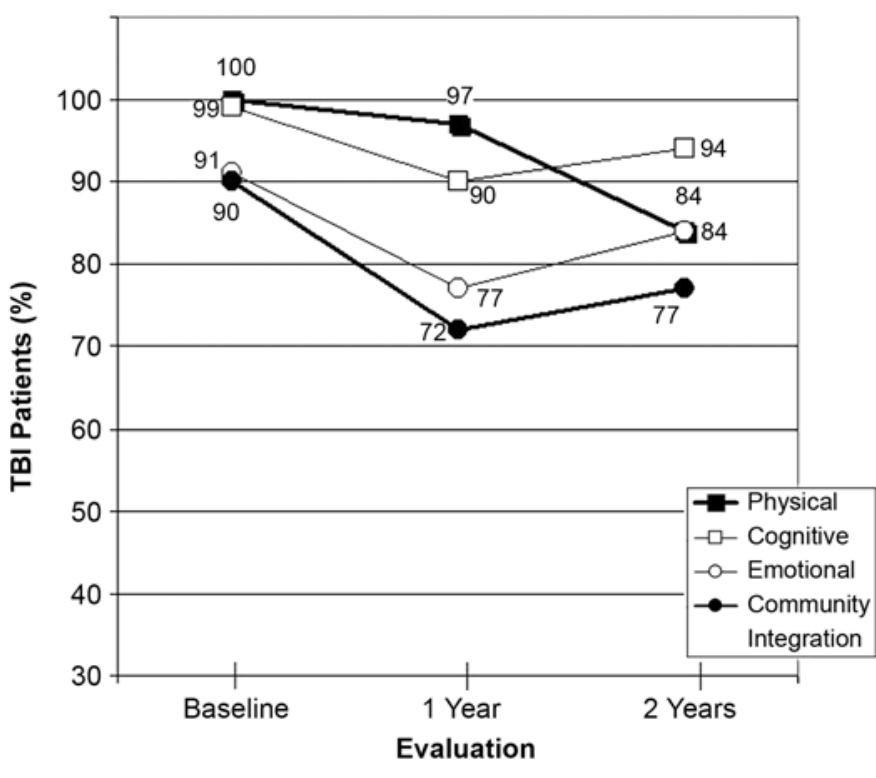

Figure 1.

Persistence of problems in traumatic brain injury (TBI) patients over a 2-year period.

which indicates gradual but steady improvement in TBI patients' physical problems over time. Similarly, problems with community integration decreased in frequency during this period from 90 to 77 percent. A noteworthy finding was the persistence of cognitive and emotional issues, with less than 10 percent decline in the frequency of these symptoms over the 2-year period. Two years after discharge, more than three-quarters of the TBI patients continued to show multiple problems.

In recent years, because of terrorism and warfare, TBI and blast-related injuries have become increasingly common in military and civilian populations [2]. Combat-related injuries are inherently stressful and place patients at elevated risk for acute stress reactions and posttraumatic stress disorder (PTSD). A previous article emphasized that many symptoms overlap between PTSD and TBI, which requires comprehensive assessment by rehabilitation and mental health professionals [3]. With this in mind, we evaluated 66 consecutive TBI patients who had completed tours of military duty in Iraq or Afghanistan. Of these 66 patients, 38 were wounded in combat and 28 sustained TBI in noncombat situations. Of those patients who sustained TBI during combat, 74 percent were victims of blast injury. Of the noncombat-injured soldiers, 71 percent were injured in motor vehicle accidents outside the war zone.

All patients completed a 13-item inventory of postconcussive and posttraumatic distress symptoms (Figure 2). Factor analysis of the inventory indicated two semi-independent clusters of symptoms (factor correlation, $r=0.31$ ). One cluster was comprised of mainly physical signs and symptoms: vision impairments, sensitivity to light or noise, dizziness, headaches, and fatigue (Figure 2, left of vertical dashed line). The second cluster consisted of mainly cognitive and emotional problems: attention/concentration deficits, memory impairment, depression, mood lability, irritability/aggression, anxiety, sleep disturbances, and posttraumatic distress (Figure 2, right of vertical dashed line).

Figure 2 compares the frequency of these 13 symptoms in our two TBI patient groups. The frequency of some symptoms did not differ greatly between the combat-injured and noncombat-injured groups. However, the overall frequency of symptoms was significantly greater in the combat-injured group than in the noncombat-injured group $(t=2.5, p=$ 0.02). On average, the noncombat-injured group had 3.8 symptoms per patient, while the combat-injured group had 5.7 symptoms per patient. This difference was largely accounted for by 4 of the 13 symptoms: vision impairments, sensitivity to light or noise, sleep disturbances, and posttraumatic distress (each $p<0.05$ ).

This analysis extends and elucidates previous observations by investigators who have reported many of the signs and symptoms in Figure 2 as sequelae of blast-related injuries. Researchers have suggested that these signs and symptoms result from the interaction of the blast wave with fluid-filled organs such as the brain and eyes, as well as from injury from heat, shrapnel, and flying debris [2,4]. Also reported are high incidence of posttraumatic headache [5], hearing loss [6], and intractable tinnitus after TBI. Because of the traumatic nature of blast-related injuries, other organs and systems are also frequently affected. Clinicians and researchers at 


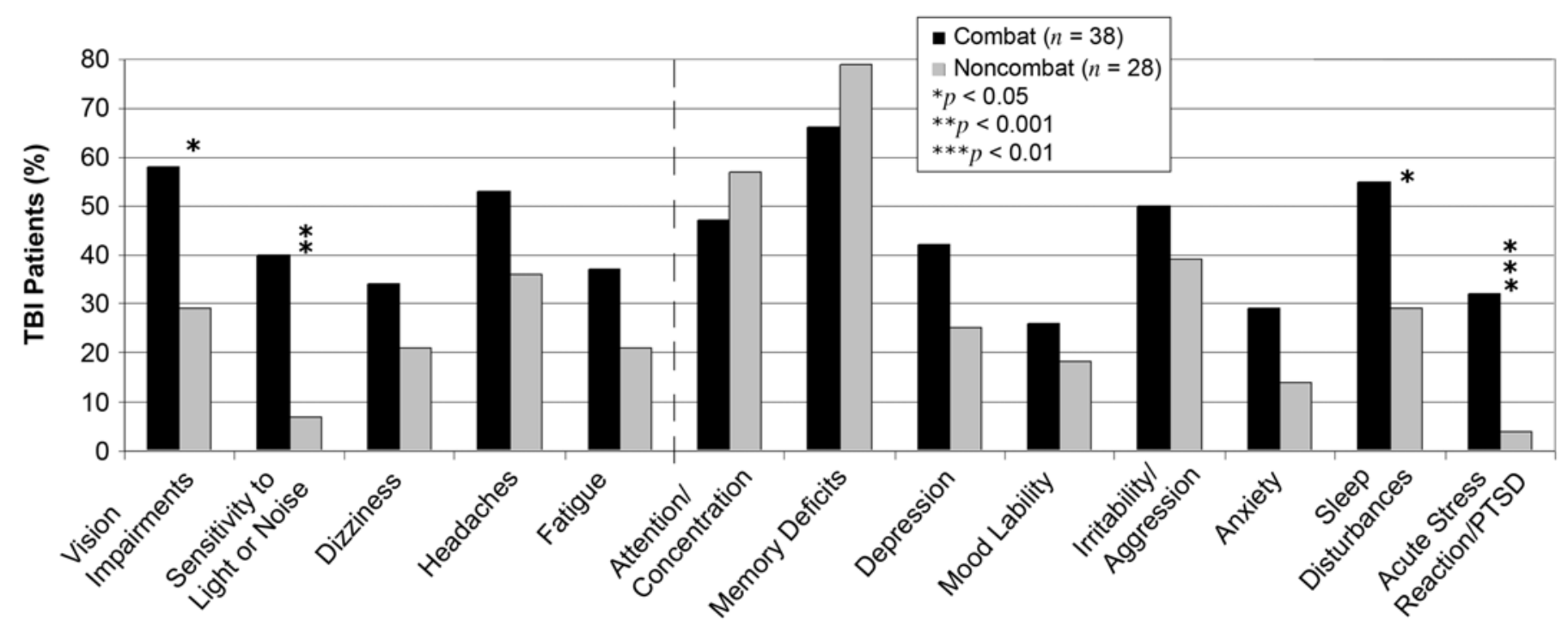

Symptoms

Figure 2.

Frequency of postconcussive symptoms in patients with combat- and noncombat-related traumatic brain injury (TBI). Factor analysis found two symptom clusters: physical (to left of vertical dashed line) and cognitive/emotional (to right of vertical dashed line). PTSD = posttraumatic stress disorder.

VA and military treatment centers are now conducting studies of combat-related polytrauma, and the clinical characteristics of this special population are beginning to unfold. For example, at our facility, we are characterizing unique clusters of symptoms and deficits that may be more likely to result from different injury mechanisms, such as blast injury, acceleration-deceleration, as well as the interaction of cognitive deficits with posttraumatic stress reactions. The ultimate goal is the development of evidencebased guidelines for treating TBI and associated trauma.

TBI patients' cognitive and emotional deficits are known to decrease their capacity and initiative to seek appropriate care on their own. Concerned families and clinicians have discussed mandatory annual follow-ups for this patient population. Undoubtedly, because of their continuing symptoms, deficits, and functional difficulties, TBI patients who do not receive coordinated care are likely to suffer significantly reduced quality of life. We found that patients who did not return for follow-up had as many physical problems at baseline as those who did return. Fur- thermore, the nonreturnees had more emotional problems at baseline. This highlights the need for standardized methods to guide TBI patients, their families, caregivers, and clinicians (especially PM\&R, mental health, and primary-care professionals) throughout the continuum of care for these frequently "invisible" injuries.

To deliver coordinated care for our returning service members, the VA recently established four Level I Polytrauma Rehabilitation Centers (PRCs) at the DVBIC's designated VA sites, Level II PRCs at each of the 21 regional Veterans Integrated Service Networks, as well as a multitude of local Level III and IV PRCs across the nation. Currently, clinicians and researchers in this network are evaluating several approaches to ensure more effective healthcare delivery, such as standardizing patients' records and treatment plans and centralizing these into a portable, electronic, code-protected format that complies with the Health Insurance Portability and Accountability Act (HIPAA). We hope these efforts will provide a model of proactive care for patients with TBI and polytrauma and enhance 
standards of practice within the VA, as well as in our non-VA healthcare systems.

\section{ACKNOWLEDGMENTS}

We gratefully acknowledge Dr. Deborah Warden, Dr. Warren Lux, Dr. Karen Schwab, Dr. Nina Sayer, Dr. Jenny Kiratli, Dr. Elaine Date, Dr. Stephen EzejiOkoye, Dr. Lawrence Leung, Dr. Joseph Bleiberg, Dr. Dennis Reeves, Dr. Heidi Queen, Ms. Gretchen Stephens, Ms. Jill Storms, Ms. Marie Dahdah, Mrs. Lisa Freeman, Dr. Darryl Thomander, and Dr. Lucille Beck for their support.

This editorial does not represent the opinions of the Department of Veterans Affairs, Department of Defense, Defense and Veterans Brain Injury Center, or the U.S. Government.

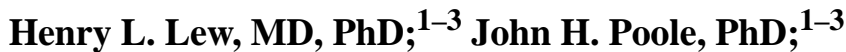
Sylvia B. Guillory, BS; ${ }^{2}$ Rose Marie Salerno, RN; ${ }^{2-3}$ Gregory Leskin, $\mathrm{PhD} ;{ }^{2}$ Barbara Sigford, $\mathrm{MD}, \mathrm{PhD}^{3-4}$

${ }^{1}$ Stanford University School of Medicine, Division of Physical Medicine and Rehabilitation; ${ }^{2}$ VA Palo Alto Health Care System; ${ }^{3}$ Defense and Veterans Brain Injury Center; ${ }^{4}$ VA Health Care System, Minneapolis, Minnesota

Email: henry.lew@va.gov

\section{REFERENCES}

1. Centers for Disease Control and Prevention. Incidence rates of hospitalization related to traumatic brain injury-12 states, 2002. MMWR Morb Mortal Wkly Rep. 2006; 55(8):201-4. [PMID: 16511440]

2. Lew HL. Rehabilitation needs of an increasing population of patients: Traumatic brain injury, polytrauma, and blast-related injuries. J Rehabil Res Dev. 2005;42(4):xiii-xvi. [PMID: 16320135]

3. Lew HL, Poole JH, Alvarez S, Moore W. Soldiers with occult traumatic brain injury. Am J Phys Med Rehabil. 2005;84(6):393-98. [PMID: 15905652]

4. Mayorga MA. The pathology of primary blast overpressure injury. Toxicology. 1997;121(1):17-28. [PMID: 9217312]

5. Lew HL, Lin P, Fu J, Wang S, Clark D, Walker W. Characteristics and treatment of headache after traumatic brain injury. Am J Phys Med Rehabil. 2006; 86(7):619-27.

6. Lew HL, Lee EH, Miyoshi Y, Chang DG, Date ES, Jerger JF. Brainstem auditory-evoked potentials as an objective tool for evaluating hearing dysfunction in traumatic brain injury. Am J Phys Med Rehabil. 2004;83(3):210-15.[PMID: 15043356]

DOI: 10.1682/JRRD.2006.05.0054 